\title{
Abstracts of the Annual Scientific Meeting of the Association of Clinical Cytogeneticists held on 30 June to 2 July 1987 at Wills Hall, University of Bristol
}

\author{
A case of diploid/triploid chimerism \\ P R MILleR, C A PARKIN, N FRASER, AND J L WATT \\ Regional Cytogenetics Unit, Maternity Hospital, Birming- \\ ham.
}

The finding of both diploid and triploid cell lines has been reported previously in patients with hypomelanosis of Ito. In such subjects, this apparent 'mosaicism' is thought to be confined to the skin since their blood lymphocytes have a normal diploid karyotype. Study of cytogenetic and DNA polymorphisms has revealed no evidence of chimerism in such cases to date. A case is presented in which there are pigmentary changes to the skin along the lines of Blaschko with a diploid blood lymphocyte karyotype and diploid/ triploid skin. In view of there being a sex mismatch between the skin cell lines $(46, \mathrm{XX} / 69, \mathrm{XXY})$, it is postulated that this case represents a true chimera. The fact that this child also has an ovotestis further suggests that the chimerism may be present in other tissues. Possible mechanisms for the origin of the chimerism are discussed. The potential benefit of cytogenetic study in patients with skin pigmentation changes is examined with regard to differentiating between monogenic disorders and cases of chimerism for the purposes of genetic counselling.

Mosaic partial monosomy $15 q$ from a $15 ; 16$ translocation M A C RIDLER, P KIRTON, J K BARBER, AND M D'A CRAWFURD Kennedy Galton Centre, Harperbury Hospital, Radlett, Herts.

Amniotic cell culture from a 38 year old woman showed a de novo mosaic $46, \mathrm{XX}, \mathrm{t}(15 ; 16)(\mathrm{q} 14 ; \mathrm{p} 13 \cdot 3) / 45, \mathrm{XX}$, $-15,-16,+\operatorname{der}(16) t(15 ; 16)(\mathrm{q} 14 ; \mathrm{p} 13 \cdot 3)$. The $\operatorname{der}(15)$ chromosome, comprising $15 \mathrm{pter} \rightarrow \mathrm{q} 14$, was missing in approximately $60 \%$ of the cells. The mosaicism might have been an artefact and fetal blood sampling and high resolution ultrasound scanning was recommended. The parents were advised of the relative risks and opted for a TOP without further investigation. The fetus showed minor dysmorphic features and culture of fetal blood confirmed the mosaic karyotype. Interpretation and counselling is difficult in this type of case and parents should be fully advised of relative risks before being subjected to the delays and trauma of extended investigations.

\author{
Anthropometric studies in the fragile $X$ syndrome \\ CAROL ENGLISH \\ Department of Human Genetics, Newcastle upon Tyne. $\vec{\infty}$
}

A series of 46 anthropometric measurements was obtained? from 365 ambulatory adult mentally retarded males resident in an institution in Northumberland. Theyo comprised 279 with a normal karyotype, 29 with the fragile$\mathrm{X}$, and 57 with other assorted abnormal karyotypes (excluding Down's syndrome). The males with the normak and assorted abnormal karyotypes were pooled, since their $\overrightarrow{0}$ measurements were not significantly different, and compared with the fragile $X$ males. The two groups had $a^{\circ}$ similar physique, but the fragile $\mathrm{X}$ males were significantlyo larger overall. However, the hands and feet of the fragile $\mathrm{X}$ males were proportionally longer and wider; their heads $\bar{\partial}$ were of greater width and circumference and they had longer, thinner faces with the extra length caused by an elongation of the jaw. These quantitative findings supported the presence of physical characteristics previouslyō described after quantitative observations. All of the fragile 3 $\mathrm{X}$ males were identified correctly by discriminant analysis on the basis of 20 measurements.

\section{A fertile mule and hinny in China}

A C CHANDLEY, RONG RHUIZHANG, S MCBEATH, AND TAN PING PING

MRC Clinical and Population Cytogenetics Unit, Western General Hospital, Edinburgh, and Institute of Genetics? Academia Sinica, Beijing, China.

Anecdotal reports of fertility in female mules (mare $\times$ jack donkey) and hinnies (jenny donkey $\times$ stallion) hav appeared in published reports over the years, but scientist $\$$ have generally regarded them with scepticism. The faco that these hybrids can come into oestrous and ovulate makes fertility a possibility, given that opportunity fo mating arises. In China, where mules are bred extensivelys for work on the farms, a fertile female mule and a fertile female hinny have now been verified by chromosomat investigation. Each had mated with a donkey and pro duced a female foal. The foals show unique hybri\& karyotypes different from the mule or hinny and differen from each other. 
The use of cytogenetic techniques and $Y$ specific DNA probes to investigate a suspected $(Y ; 14)$ translocation product

A CLARK

Centre for Human Genetics, Sheffield.

Cytogenetic investigation of a woman following repeated miscarriages revealed a $14 \mathrm{q}+$ marker chromosome. Cytogenetic techniques, including anaphase studies, $\mathrm{C}$ banding, and DA-DAPI staining suggested the chromosome was the product of a $(Y ; 14)$ translocation, but did not permit the assignment of a $\mathrm{Y}$ chromosome breakpoint. Family studies have demonstrated unbalanced karyotypes involving this product in five members of three generations, the only adult male carrier being referred independently as a result of his infertility. His sperm exhibited $100 \%$ abnormal forms and no motility. DNA studies using a panel of $Y$ specific probes have been used to demonstrate the extent of $\mathrm{Y}$ chromosome involvement.

\section{Analysis of the origin of Turner's syndrome using DNA probes \\ A REDHA, L A PIRRIT, E BOYD, AND J M CONNOR \\ Duncan Guthrie Institute of Medical Genetics, Glasgow.}

Thirteen nuclear families with a child or fetus with Turner's syndrome were studied using DNA restriction fragment length polymorphisms. The karyotypes of the probands were as follows: five had 45, X karyotype, four were mosaics with $45, \mathrm{X} / 46, \mathrm{Xr}(\mathrm{X})$, two were mosaics $45, X / 46, X i(X q)$, one was mosaic $45, X / 46, X X$, and one was $46, X i(X q)$. Of the $45, X$ probands, two were children and there were three terminations following prenatal diagnosis. Using the DNA probe St14-1 (DXS52) which maps to $\mathrm{Xq} 28$, parental origin of non-disjunction could be determined in 10 cases, and in the $45, \mathrm{X}$ cases loss of the paternal homologue was more frequent than the loss of the maternal X. By simultaneous use of further DNA probes from the proximal long arm and $\mathrm{Xp}$ it was possible to determine the timing and origin of mosaicism and isochromosome formation.

Localisation of chromosome breakpoints in ataxia telangiectasia lymphocytes using in situ hybridisation

A M R TAYLOR, S V BUTTERWORTH, R HOLLIS, A HEPPELL, A A KENNAUGH, AND J A METCALF

Department of Cancer Studies, Medical School, Birmingham.

Various chromosomal translocations have been described in peripheral lymphocytes from patients with ataxia telangiectasia often involving the sites of the immunoglobulin superfamily genes (Aurias et al, Hum Genet 1986;72:210). Clones of cells with some of these translocations may appear in the blood, most being very small. We have studied two large non-leukaemic clones $(70 \%$ of $T$ cells) with translocations $t(14 ; 14)(q 11 ; q 32)$ and $t(X ; 14)$ (q28;q11) respectively. Using in situ hybridisation of probes on the $t(14 ; 14)$ clone we have shown that the $14 \mathrm{q} 32$ breakpoint lies outside the IgH locus and proximal to it with respect to the centromere. The $14 q 11$ breakpoint in the homologous 14 splits the T cell receptor $\alpha$ chain locus with the constant region being translocated to the $14 \mathrm{q}+$ chromosome leaving the variable region on the $14 \mathrm{q}-$ chromosome (Kennaugh et al, Hum Genet 1986;73:254, and unpublished results). In the $t(X ; 14)$ the constant region of the $\alpha$ chain locus is translocated to $\mathrm{Xq} 28$, and although a reciprocal translocation is likely from $X$ to 14 , the breakpoint appears distal to the site of the RFLP St14 and to the G6PD gene. Both clones therefore appear to involve translocation of a single $T$ cell receptor gene to the site of an unidentified gene. Involvement of the $\alpha$ chain gene may be required for successful non-malignant $T$ cell proliferation and further genetic change is required for transformation to full malignancy.

\section{Molecular analysis of the fragile $X$ syndrome}

K E DAVIES, M PATTERSON, M BELL, AND S J KENWRICK Nuffield Department of Clinical Medicine, John Radcliffe Hospital, Oxford.

The fragile $\mathrm{X}$ syndrome is associated with a fragile site at $\mathrm{Xq} 27 \cdot 3$ and is a disease that affects approximately 1 in 1100 males. The disorder shows several unusual features in that there is a high rate of expression in female heterozygotes and normal males have been found to transmit the mutation. A full understanding of the molecular basis of these observations awaits the isolation of DNA sequences within the region of the fragile site. The DNA probes localised to Xq27 $\rightarrow$ Xqter that bridge the fragile site have been used to construct a genetic map of the region. It is evident that the genetic distances are quite large compared to the physical distances and that genetic heterogeneity exists between affected families. Long range restriction mapping by pulsed field gel electrophoresis analysis is invaluable for the localisation of new genes and the probing of the fragile site region. In particular, clusters of sites for rarely cutting restriction enzymes can be identified which represent good candidates for HTF islands. HTF islands are associated with expressed sequences in the mammalian genome and therefore represent a method by which we can identify candidate genes for this particular disorder.

Biotin labelled in situ hybridisation maps 1 kb single copy gene

J A VAN DEN BERGHE, J GARSON, AND S MALCOLM Institute of Child Health, London.

A method is described for the fine mapping of single copy genes using biotin labelled DNA probes and an alkaline phosphatase detection system. The technique may be used on slides which have been previously trypsin $\mathrm{G}$ banded and mounted, permitting the use of routine clinical cytogenetic material, possibly archival. Other advantages over radioactive methods include safety, low background, accuracy, and speed, development of label occurring within one to four hours as opposed to two to three weeks for ${ }^{3} \mathrm{H}$ labelled probes. The higher resolution obtained with this method has allowed a number of single copy genes, with insert sizes as small as $1 \mathrm{~kb}$, to be mapped with precision. 
I'he technique would be of use in the identification of marker or supernumerary chromosomes and the appearance of label in interphase nuclei may allow detection of sex or exclusion of trisomy in non-dividing prenatal samples.

The effect of methotrexate on the quantity and quality of metaphases in ANLL bone marrow cultures J J P CUNNINGHAM, A M POTTER, AND A E WATMORE Centre for Human Genetics, Sheffield.

Analysis of bone marrow cultures from leukaemic patients is often impaired by chromosomal contraction and low mitotic index (MI). The well known cytogenetic technique of methotrexate (MTX) block/thymidine release synchronisation, considered to improve mitotic yield and chromosome length, has been examined in detail. Acute non-lymphocytic leukaemic (ANLL) bone marrows were cultured, according to the standard technique, and incubated $+/-$ MTX before release. No significant difference was detectable in either MI or chromosome length. Cell division in the presence of MTX was examined by harvesting after 17 hours with MTX without releasing and no significant block was evident. Pretreatment incubation times and duration of colcemid exposure were also examined. No significant change in cell division or improvement in chromosome quality attributable to MTX was detected, and its suitability for bone marrow synchronisation is questioned. Results indicating improved chromosome length after ethidium bromide treatment will be discussed.

Acute megakaryoblastic leukaemia in childhood. The role of chromosome 21

CLARE KITCHEN, A O M WILKIE, AND R T HOWELL SW Regional Cytogenetics Centre, Bristol.

A case of acute megakaryoblastic leukaemia in a two year old girl with Down's syndrome is presented. This rarely reported malignancy has been described more frequently in children with Down's syndrome. Normal children with this leukaemia frequently have acquired trisomy 21 . The leukaemic cells in the present patient have a previously unreported chromosomal change interpreted as dic $(5 ; 7)$ (p12;p11·1).

How can chromosome 9 or 22 involvement be hidden in aberrant translocations in chronic myelogenous leukaemia (CML)

J L HURET, J L BRESSON, AND J TANZER

Hôpital Jean Bernard, Poitiers, France.

Besides two personal cases $(\mathrm{t}(4 ; 22)(\mathrm{p} 16 ; \mathrm{q} 11)$ and $\mathrm{t}(12 ; 22)$ (p13;q11)), we collected 78 such translocations $(t(M ; 22)$ and $t(M ; 9)$ ) involving chromosome 9 or 22 and an extra chromosome ( $M$ and/or $\left.\mathbf{M}^{\prime}\right)$. The breakpoint on $M$ was defined as telomeric (T) or not (NT). In a $t(M ; 22) T$, number 9 involvement could be masked if, in a circular translocation $\mathrm{t}(\mathrm{M} ; 9 ; 22)$, the 9 telomere is replaced by a telomere of $\mathbf{M}$ of the same size and grey level. Abl would be translocated next to Bcr as usual in CML. In a
$t(M ; 22) / N T$, a short gene sequence from chromosome $9 \overrightarrow{\overline{\text { जे }}}$ would seem of normal size. In a $t(M ; 9) / N T$, a short gene sequence including $\mathrm{Bcr}$ would be translocated onto $M \frac{\bar{O}}{\sigma}$ before 9 telomere including $\mathrm{Abl}$. These last two con- $\overline{\bar{C}}$ figurations have already been ascertained by in situ $\vec{\Phi}$ hybridisation. In a CML $\mathrm{Ph}-$, only a short gene sequence $\varrho$ including $\mathrm{Abl}$ would be translocated next to Bcr. Finally, six cases of $t(M ; 9), t\left(M^{\prime} ; 22\right)$ have been published, five of $\rightarrow$ them with a telomeric breakpoint on $\mathbf{M}^{\prime}, \mathbf{M}^{\prime}$ telomere? could be translocated onto $M$ without being detectable, $a \overrightarrow{\vec{\Delta}}$ part of $M$ onto 9 as detected, 9 telomere onto 22 as usual in CML, and 22q12 $\rightarrow$ qter onto $\mathbf{M}^{\prime}$ as detected, in a circular translocation $t\left(M ; 9 ; 22, M^{\prime}\right)$. Similarly, the usual $t(9 ; 22)$ قै might occasionally be a $t(M ; 9 ; 22)$ where $M$ involvement is hidden.

Myelodysplastic syndromes. Clonal origin and evolution J G MUFTI King's College School of Medicine, London.

The myelodysplastic syndromes (MDS) constitute a heterogeneous group of clonal disorders, arising from a multipotent haemopoietic progenitor, which share a leukaemic $\vec{\theta}$ propensity, $30 \%$ of cases culminating in acute myeloid $\infty_{\infty}$ leukaemia (AML). Non-random chromosomal abnormalities are documented in 30 to $79 \%$ of cases and karyotypic evolution is usually associated with a clinical progression of the disease. Their pathogenesis probably entails multiple $\bar{\partial}$ steps, phenotypic progression being determined by either expansion or evolution of the abnormal clone. The clonal $\mathbb{Q}$ origin of certain cases of de novo AML is analogous to that of MDS and evidence that they share a common pathogenesis and distinct biological characteristics is beginning to emerge. However, difficulties in early diagnosis remain, since morphological abnormalities may be subtle and karyotypic abnormalities may be absent. In view of this, alternative methods of diagnosis of clonal expansion which would complement chromosomal analysis are being $\delta$ attempted. Preliminary results of the use of sex linked 3 DNA polymorphism at the hypoxanthine guanine phosphoribosyl transferase (HPRT) locus are encouraging. The $\frac{0}{3}$ application of techniques currently in use in molecular biology may help us understand the pathogenesis of this disease which has been called a human model for the study of leukaemias.

Cytogenetic and DNA polymorphism studies in leukaemia patients having bone marrow transplants CHRISTINE HARRISON

Christie Hospital and Holt Radium Institute, Manchester.

The value of cytogenetic and DNA polymorphisms as early indicators of engraftment and relapse after bone marrow transplantation is being assessed. Leukaemic cells are often marked by chromosome abnormalities, which usually $\frac{\vec{\Phi}}{\mathbb{D}}$ reappear in relapse. In the absence of chromosome $\stackrel{\Phi}{\Phi}$

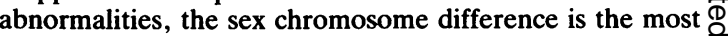
widely used technique to document engraftment. When 
the transplant is made between subjects of the same sex, normal chromosome variants may be helpful. A range of techniques have been used to demonstrate these, including SEM. In the absence of striking chromosomal variations, restriction fragment length polymorphisms (RFLPs), which indicate variation in the primary DNA sequence, may be used. A number of transplant cases have been studied using cytogenetic and DNA techniques. The findings will be presented and the relative values of the two procedures in monitoring transplantation will be discussed.

Interstitial deletion of chromosome 13 in three cases with retinoblastoma: further localisation of the $R b 1$ gene $S$ H ROBERTS AND M LITTLER

Institute of Medical Genetics, University Hospital of Wales, Cardiff.

Retinoblastoma, a childhood tumour, occurs with a frequency of about 1 in 10 to 20000 . The majority are sporadic but about $5 \%$ have an autosomal dominant mode of inheritance with $90 \%$ penetrance. Some 5 to $10 \%$ of sporadic cases have a cytogenetic imbalance, which is usually the result of an interstitial deletion. We report three further cases of interstitial deletions of chromosome 13, $\operatorname{del}(13)(\mathrm{q} 13 \cdot 2 \mathrm{q} 22), \operatorname{del}(13)(\mathrm{q} 13 \cdot 3 \mathrm{q} 22)$, and $\operatorname{del}(13)$ $(\mathrm{q} 14 \cdot 3 \mathrm{q} 22)$, associated with retinoblastoma. The tumour was unilateral in two cases and bilateral in one. The levels of the linked enzyme esterase D were $57 \%, 54 \%$, and $64 \%$ respectively. One deletion case was ascertained through the retinoblastoma but the other two were ascertained through failure to thrive and dysmorphism or developmental delay. The cytogenetic findings alerted the clinicians to the possibility of a tumour in one child and early treatment avoided the resort to enucleation. Dysmorphic features were observed in all three children. The proximal breakpoint within 13q14.3 appears to exclude the genes for retinoblastoma and esterase D from $13 q 14 \cdot 1$ and $13 q 14 \cdot 2$.

\section{Are double translocations double trouble?}

$S$ M BOWSER-RILEY, $M$ J GRIFFITHS, P A FARNDON, $M R$ CREASEY, K E MARTIN, D A THOMSON, S A LARKINS, AND J L WATT

Regional Cytogenetics Unit, Maternity Hospital, Birmingham.

Double transplantation heterozygotes are rare, but need not pose more of a counselling problem than single reciprocal translocations. Nine cases of double translocations will be briefly presented and used to illustrate possible counselling guidelines for the assessment of risk of producing a liveborn abnormal child. These suggested guidelines are not based on theoretical considerations or segregation patterns, but are extrapolated from what is known empirically about segregation patterns in carriers with single reciprocal translocations. An assumption is made that there is no interference with the independent assortment of two separate exchanges, unless a common participating chromosome is involved and the possibility of an interchromosomal effect is not taken into consideration.
Prenatal diagnosis of a pure trisomy $14 / 18$ mosaic

J C K BARBER, C A TIMBERLAKE, AND M A C RIDLER Kennedy Galton Centre, Harperbury Hospital, Radlett, Herts.

Trisomy 18 was diagnosed in the fetus of a 40 year old mother of five following amniocentesis at 17 weeks' gestation. The phenotype of the fetus after TOP was consistent with this diagnosis. However, cultures of skin and chorion produced two cell lines with trisomy 14 predominant over trisomy 18 in both skin $(82 \%)$ and chorion $(65 \%)$. Subsequent re-examination of preparations from the original amniotic cell culture confirmed trisomy 14 as a minor cell line only $(4 \%)$. The possible origin of this unusual case is discussed.

\section{Prenatal diagnosis of fragile $X$ syndrome by placental} biopsy

M J MCKINLEY, L U KEARNEY, AND K NICOLAIDES

King's College School of Medicine, London.

Two out of eight male fetuses (gestation 19 to 20 weeks) at risk for fragile $\mathrm{X}$ linked mental retardation (Martin-Bell syndrome) were diagnosed as affected after cordocentesis and subsequent fetal blood culture $(4 \%$ and $21 \%$ of cells with $\mathrm{fra}(\mathrm{X})$ respectively). In both cases fra(X) was also demonstrated in placental biopsy cultures (PBC) $(0.4 \%$ and $4 \%$ of cells respectively). $\operatorname{Fra}(\mathrm{X})$ was demonstrated using methotrexate, 5-fluorodeoxyuridine (FUdR), and excess thymidine. $\mathrm{Fra}(\mathrm{X})$ expression with FUdR was not enhanced by caffeine or 5-methoxybenzamide. The use of caffeine may lead to ambiguous results.

\section{Long range restriction mapping of human chromosomes} DUNCAN SHAW

Institute of Medical Genetics, University Hospital of Wales, Cardiff.

Since molecular cloning and sequencing operates on DNA molecules of up to a few kilobases length, and since cytogenetics and family linkage studies do not provide resolution below the million base pair level, there has existed a 'resolution gap' in the range of techniques available to human geneticists. It is now possible to fill this gap using various forms of pulsed gel electrophoresis, which are capable of separating DNA in the size range 50 milobases to 10 megabases. By using these techniques in conjunction with restriction enzymes that cut human DNA very infrequently, long range maps of chromosomal regions and even whole chromosomes can be constructed, cytogenetically invisible DNA rearrangements can be detected, and strategies for isolating disease genes can be envisaged. Some preliminary data involving human chromosome 19 have been obtained.

\section{The effect of deletions on carrier status determination in} DMD

DAVID ROBINSON AND JOHN HARVEY

Regional Cytogenetics Unit, General Hospital, Salisbury.

The Wessex Regional Cytogenetics Unit is compiling a DNA register of families within the region with a history of 
cystic fibrosis, Huntington's chorea, and the muscular dystrophies for prenatal diagnosis and carrier detection. Allelic status is assessed by identification of RFLP bands on an autoradiograph. However, in certain cases results may be ambiguous. In DMD families where a deletion is shown in the proband, or if the affected family member is dead, the identification of the carrier status of female relatives may not always be possible by simple identification of RFLP bands. A case is presented where subjective assessment of allelic concentration on the basis of dose response is necessary.

Further studies on the cystic fibrosis locus BOB WILLIAMSON St Mary's Hospital Medical School, London.

By a combination of chromosome mediated gene transfer, the characterisation of cosmid contigs, the identification of HTF islands signalling coding sequences, and the use of linkage and linkage disequilibrium, a set of cloned markers has been isolated which invariably segregate with the CF mutation. The best defined of these markers identify a coding sequence which is expressed in epithelial cells, as well as three RFLPs which can be used for prenatal diagnosis and carrier determination in families with a living CF person. The disequilibrium is so marked that two of the probes can also be used for carrier exclusion. More than $50 \%$ of families have a very small risk of having a CF child based upon these criteria alone, but the associated allele is sufficiently common that carrier determination cannot yet be attempted successfully. The coding sequence is a candidate gene for the protein which is mutated to cause CF.

CVS at Guy's Hospital: diagnostic and research experience M MURER-ORLANDO, J LLERENA JR, L ZAHED, M MCGUIRE, J CROLLA, $Z$ DOCHERTY, C FEAR, AND M BOBROW Paediatric Research Unit, Guy's Hospital, London.

For the past year, our group has been working on CVS material, providing a diagnostic service mostly for the Guy's MRC CVS-amnio trial, while efforts have been made to improve the cytogenetic techniques and to understand the biology of this tissue. Diagnostic experience. We have analysed 330 cases using direct (DP) and culture (CP) protocols. Direct preparations were suitable for chromosome count, sexing, and GTG banding. Analysis of metaphases up to the 300 band stage was possible in $70 \%$ of the cases. In our experience we have encountered four situations which had to be carefully evaluated: mosaic trisomy 7 detected only in DP and not confirmed in CP, analysis of two familial rearrangements $(\mathrm{t}(4 ; 5)(\mathrm{q} 35 ; \mathrm{q} 33)$, inv or iso(13) which were only possible in $C P$, and fine rearrangement of chromosome $8 p$ detected in $\mathrm{CP}$ but missed in DP. Research experience. Direct, semi-direct, and culture protocols have been adapted to CVS material in an effort to obtain good preparations in short times. Explant cultures have been successfully harvested within 11 days. To accelerate the analysis of DP, automatic scanning has been evaluated using the Magiscan and a laboratory research precursor of Metafip. For a complete cytogenetic analysis of DP standard banding protocols have been modified, namely QFQ, RFG, CBG, DAPI and NOR. Cell cycle studies have been performed using BUdR incorporation, which have allowed us to establish protocols for first trimester prenatal diagnosis of chromo some instability disorders.

Can the 'chorionscope' help in providing a diagnostic CVSservice for a distant district hospital

ROD HOWELL AND SIMON WOOD

SW Regional Cytogenetics Centre, Bristol.

Use of the chorionscope permits direct visualisation of the site of chorion biopsy, thus reducing the dependence of the obstetrician on high resolution ultrasound equipment and dispensing with the need for immediate evaluation of thesample under the dissecting microscope. This approach, inos conjunction with rapid rail transport of the specimen to theo laboratory, is currently under evaluation and it is hoped that the provision of a diagnostic CVS service by the District Hospital at a distance from the cytogeneticsi laboratory will eventually become possible.

Pseudomosaicism in chorionic villi

NINA M GREGSON

Regional Cytogenetics Unit, General Hospital, Salisbury.

The problems of distinguishing true from false mosaicism in amniotic fluid cells are well known. Similar difficulties are sometimes encountered in cells from chorionic villi. $A$ case is described here where all cells from a semi-direct (24\% hour) culture had a complement of $48, X X,+18,+20$. In the long term culture (eight days), however, only $45 \%$ of the cells were abnormal. True mosaics for +18 are rare and should be investigated further. In fact, in this case a fetab blood sample showed a normal $46, \mathrm{XX}$ in over 100 cells examined. The pregnancy is continuing.

Evaluation of sampling procedures in cytogenetics: ani exercise in quality control

MIGUEL A DE ARCE AND SUSAN P MCMANUS Children's Hospital, Dublin.

Confirmation of the coexistence of two cell lines (mosaicism? or chimerism) in the same subject is a frequent problem for cytogeneticists who should rely for this task on soundo sampling procedures. These are often dictated by staffshortages rather than by acceptable statistical practice o Without making rigid suggestions, we propose a simplên way to deal with this problem in its various forms. We have plotted sample size against frequency of second cell line tow obtain a given probability of detecting $0,1,2$, or 3 cells of the less abundant line. This suggests an iterative procedureo to deal with possible mosaicism detectable in blood cells and several situations will be discussed. A similar method? is described to detect genuine fetal mosaicism as it would appear in amniotic fluids harvested in situ or by the flask 6 technique, to distinguish it from pseudomosaicism. For the diagnosis of fragile $X$, we show the power of sampling plans $50(0 \cdot 1), 50(1 \cdot 2), 100(0 \cdot 1), 100(1 \cdot 2), 200(0 \cdot 1)$, and 200(1.2) as defined in British Standard BS 6001, 1972. 